IFT-UAM/CSIC-11-104

FTUAM-11-67

\title{
Lepton flavor violation in minimal flavor violation extensions of the seesaw
}

\author{
Enrico Nardi \\ INFN, Laboratori Nazionali di Frascati,C.P. 13, 100044 Frascati, Italy. \\ and: \\ Departamento de Física Teórica, C-XI, Facultad de Ciencias, \\ Universidad Autónoma de Madrid, C.U. Cantoblanco, 28049 Madrid, Spain \\ and: \\ Instituto de Física Teórica UAM/CSIC, Nicolas Cabrera 15, C.U. Cantoblanco, 28049 Madrid, Spain
}

\begin{abstract}
A minimal flavour violation hypothesis for leptons can be implemented essentially in two ways that are compatible with a type-I seesaw structure with three heavy singlet neutrinos $N$, and that satisfy the requirement of being predictive, in the sense that all lepton flavor violating (LFV) effects can be expressed in terms of low energy observables. The first realization is $C P$ conserving and is based on the flavor group $S U(3)_{e} \times S U(3)_{\ell} \times O(3)_{N}$ (being $e$ and $\ell$ the $S U(2)$ singlet and doublet leptons). The second realization allows for $C P$ violation and is based on $S U(3)_{e} \times S U(3)_{\ell+N}$. I review the main features of the two schemes and point out their different implications for LFV observables.
\end{abstract}

Keywords: Neutrino Physics, Global Symmetries, Rare Decays

\section{Introduction}

The assumption that the sources of breaking of the flavour symmetry present in the standard model (SM) Lagrangian determine completely the structure of flavour symmetry breaking also beyond the SM, is commonly referred to as the Minimal Flavour Violation (MFV) hypothesis [1-3]. In the quark sector there is a unique way to implement MFV: the two quark SM Yukawa couplings are identified as the only relevant breaking terms of the $S U(3)^{3}$ quark-flavour symmetry [3]. For the lepton sector the same is not true: the SM cannot accommodate Lepton Flavour Violation (LFV) because there is a single set of Yukawa couplings (those of the charged leptons) that can always be brought into diagonal form by rotating the three $S U(2)_{L}$-doublets $\ell_{\alpha}$ and the three right-handed $(\mathrm{RH})$ $S U(2)_{L}$-singlets $e_{\alpha}(\alpha=e, \mu, \tau)$. However, with the discovery of neutrino oscillation it has been clearly established that lepton flavor is not conserved. It is then

\footnotetext{
${ }^{4}$ Invited talk at the Workshop on $\mathrm{e}^{+} \mathrm{e}^{-}$Collisions from $\Phi$ to $\Psi$ (PHIPSI08), September 19-22, 2011, Novosibirsk, Russia. Based in part on work done in collaboration with R. Alonso, G. Isidori, L. Merlo and L. A. Muñoz.
}

interesting to extend the MFV hypothesis to the lepton sector (MLFV) by starting from a Lagrangian able to describe the observed LFV in neutrino oscillations. The problem is that we do not know which physics beyond the SM is responsible for these effects, and different generalizations of the SM yield different formulations of the MLFV hypothesis.

\section{Minimal effective theories for the seesaw}

A theoretically very appealing way to extend the SM to a dynamical model that can account for strongly suppressed neutrino masses is the type-I seesaw, where it is assumed that in addition to the SM leptons $(\ell$ and $e$ ) at high energies there is at least another set of dynamical fields carrying lepton flavour: three SM singlets heavy Majorana neutrinos $N_{i}$. The gauge invariant kinetic terms for the lepton fields $\ell_{\alpha}, e_{\alpha}$ and $N_{i}$ is:

$$
\mathcal{L}_{\text {Kin }}=\bar{\ell}_{\alpha} \not D_{\ell} \ell_{\alpha}+\bar{e}_{\alpha} \not D_{e} e_{\alpha}+\bar{N}_{i} \not \partial N_{i},
$$

where $D_{\ell}, D_{e}$ denote covariant derivatives. The largest group of flavour transformations that leaves $\mathcal{L}_{\text {Kin }}$ invariant is $\mathcal{G}=U(3)_{\ell} \times U(3)_{N} \times U(3)_{e}$. We assume that $\mathcal{G}$, 
or some subgroup of $\mathcal{G}$, is the relevant group of flavour transformations, and we require that the only symmetrybreaking terms can be identified with the parameters appearing in the seesaw Lagrangian, that is:

$$
\begin{aligned}
-\mathcal{L}_{\text {seesaw }} & =\epsilon_{e} \bar{\ell}_{\alpha} Y_{e}^{\alpha \beta} e_{\beta} H+\epsilon_{v} \bar{\ell}_{\alpha} Y_{v}^{\alpha j} N_{j} \tilde{H} \\
& +\frac{1}{2} \epsilon_{v}^{2} \mu_{L} \bar{N}_{i}^{c} Y_{M}^{i j} N_{j}+\text { h.c.. }
\end{aligned}
$$

The symmetry group can be decomposed as $\mathcal{G}=$ $U(1)_{Y} \times U(1)_{L} \times U(1)_{R} \times \mathcal{G}_{F}$ where $U(1)_{Y}$ and $U(1)_{L}$ correspond to hypercharge (that remains unbroken) and to total lepton number, respectively; $U(1)_{R}$ can be identified either with $U(1)_{e}$ or with $U(1)_{N}$, corresponding respectively to global phase rotations of $e$ or $N$, and

$$
\mathcal{G}_{F}=S U(3)_{\ell} \times S U(3)_{N} \times S U(3)_{e},
$$

is the flavour group, broken at some large scale $\Lambda_{F} \gg$ $\mathrm{TeV}$. Formal invariance of $\mathcal{L}_{\text {seesaw }}$ under $\mathcal{G}_{F}$ is recovered by promoting the Lagrangian parameters to spurions transforming as:

$$
Y_{v} \sim(3, \overline{3}, 1) ; \quad Y_{M} \sim(1, \overline{6}, 1) ; \quad Y_{e} \sim(3,1, \overline{3}) .
$$

As regards the two broken Abelian factors, $U(1)_{L}$ is broken (by two units) by $\mu_{L}$, that is a spurion with dimension of a mass, while $U(1)_{R}$ is broken by a dimensionless spurion $\epsilon_{R}$, where $\epsilon_{R}$ denotes $\epsilon_{e}$ or $\epsilon_{v}$.

By itself, the Lagrangian eq. (2) induces LFV effects for the charged leptons that are well below $O\left(10^{-50}\right)$, and thus unobservable. However, a theoretical prejudice states that there is new physics at the $\mathrm{TeV}$ scale, since this is needed to cure the SM naturalness problem. It is then reasonable to assume that at some scale $\Lambda_{N P} \ll \Lambda_{F}, \mu_{L}$, presumably around or somewhat above the electroweak scale, other states carrying flavour exist. Integrating out these heavy degrees of freedom, as well as the heavy RH neutrinos with masses $\epsilon_{v}^{2} \mu_{L}>\mathrm{TeV}$, at $E \ll \mathrm{TeV}$ we obtain an effective Lagrangian of the form:

$$
\mathcal{L}_{\text {eff }}=\mathcal{L}_{\mathrm{SM}}+\mathcal{L}_{D 5}^{\text {seesaw }}+\frac{1}{\Lambda_{N P}^{2}} \sum_{i} c_{i} O_{i}^{(6)}+\ldots
$$

$\mathcal{L}_{D 5}^{\text {seesaw }}$ is the Weinberg operator [4] that depends on the spurions (see eq. (6)). $O_{i}^{(6)}$ denote generic dimensionssix operators written in terms of the SM fields and of the spurions, and the dots denote higher dimension operators. Dimensions-six operators involving only the SM fields conserve $B-L$ [4], and since we have not introduced (dangerous) sources of $B$ violation, then the operators $O_{i}^{(6)}$ must conserve separately $L$. This is the reason why the scale $\Lambda_{N P}$ can be substantially lower than $\Lambda_{F}$ and $\mu_{L}$. Note also that $U(1)_{N}$ breaking and $\epsilon_{v}$ only affect the RH neutrino masses, without affecting in any way the Weinberg operator (see eq. (6)). As far as the flavour structure of the operators $O_{i}^{(6)}$ is concerned, the assumptions about $\mathcal{G}_{F}$ breaking imply the following:

I. Once the transformation properties of the spurions eq. (4) and of the fields are taken into account, all $O_{i}^{(6)}$ must be formally invariant under $\mathcal{G}_{F}$.

This condition alone is not sufficient to obtain an effective theory that is predictive, since the flavour structure of $Y_{v}, Y_{M}$ and $Y_{e}$ cannot be determined from low-energy data alone [5]. A predictive MLFV formulation must satisfy an additional working hypothesis:

II. The spurions flavour structure must be reconstructable from low energy observables, namely the light neutrino masses and the PMNS mixing matrix.

The only way this second hypothesis can be satisfied is by restricting the form of the spurions $Y_{i}$ in such a way that the relevant LFV combinations will depend on a reduced number of parameters. This can be obtained by assuming that the flavour symmetry corresponds to a subgroup of $\mathcal{G}_{F}$, rather than to the full flavour group.

\section{1. $\boldsymbol{U}(\mathbf{1})_{\boldsymbol{R}}$ breaking and size of the LFV effects}

Before analyzing the possible subgroups of $\mathcal{G}_{F}$ yielding predictive frameworks, let us discuss the connection between the overall size of the LFV effects and the breaking of $U(1)_{R}$. The explicit structure of $\mathcal{L}_{D 5}^{\text {seesaw }}$ and the corresponding light neutrino mass matrix are

$$
\begin{aligned}
\mathcal{L}_{D 5}^{\text {seesaw }} & =\frac{1}{\mu_{L}}(\bar{\ell} \tilde{H}) Y_{v} \frac{1}{Y_{M}} Y_{v}^{T}\left(\tilde{H}^{T} \ell^{c}\right), \\
\Longrightarrow \quad m_{v}^{\dagger} & =\frac{v^{2}}{\mu_{L}} Y_{v} \frac{1}{Y_{M}} Y_{v}^{T}=U \mathbf{m}_{v} U^{T},
\end{aligned}
$$

where $v$ is the Higgs vacuum expectation value, $U$ is the PMNS matrix and $\mathbf{m}_{v}=\operatorname{diag}\left(m_{v_{1}}, m_{v_{2}}, m_{v_{3}}\right)$. Note that since $\mathcal{L}_{D 5}^{\text {seesaw }}$ does not break $U(1)_{R}$, the overall size of $\mathbf{m}_{v}$ depends only on the lepton-number violating scale $\mu_{L}$, but not on $\epsilon_{e, v}$. Without loss of generality we can rotate $Y_{e}$ and $Y_{M}$ to a diagonal basis. In terms of mass eigenvalues the diagonal entries can be written as:

$$
\left(Y_{e}\right)_{\alpha \alpha}=\frac{1}{\epsilon_{e} v} m_{\alpha}, \quad\left(Y_{M}\right)_{i i}=\frac{1}{\epsilon_{v}^{2} \mu_{L}} M_{i} .
$$

This shows that the overall size of $Y_{e}$ and $Y_{M}$ is controlled by the Abelian spurions (the same is true for $Y_{v}$ ). A natural choice for their size is such that the entries 
in the $Y_{i}$ matrices are of $O(1)$. Considering the lightneutrino mass matrix eq. (7) it can be seen how this choice points to a very large $L$-breaking scale

$$
\mu_{L} \sim v^{2} / \sqrt{\Delta m_{\mathrm{atm}}^{2}} \approx 6 \times 10^{14} \mathrm{GeV} .
$$

In the case when $U(1)_{R}=U(1)_{N}$ however, we are free to assume $\epsilon_{v} \ll 1$ as would naturally result from an approximate $U(1)_{N}$ symmetry. In this case, in spite of the large values of $\mu_{L}$, the RH neutrinos could have much smaller masses, possibly within the reach of future experiments which, from the phenomenological point of view, this represents a very interesting possibility [6].

\subsection{Two predictive cases}

The dimension-six LFV operators $O_{i}^{(6)}$ are invariant under $U(1)_{L}$ and $U(1)_{N}$, but break $\mathcal{G}_{F}$ through various spurions combinations, like for example:

$$
\Delta_{8}^{(1)}=Y_{v} Y_{v}^{\dagger} ; \quad \Delta_{6}=Y_{v} Y_{M}^{\dagger} Y_{v}^{T} ; \quad \Delta_{8}^{(2)}=Y_{v} Y_{M}^{\dagger} Y_{M} Y_{v}^{\dagger}
$$

In the absence of further assumptions, the $\Delta$ 's cannot be determined in terms of $U$ and $\mathbf{m}_{v}$. To obtain predictive frameworks basically two different criteria can be adopted, that correspond to assume that in a given basis either $Y_{M}$ or $Y_{v}$ are proportional to the identity matrix in flavour space $I_{3 \times 3}[5,6]$. Both these criteria have the property of being natural in the sense that they can be formulated in terms of symmetry hypotheses, that is by choosing as flavour symmetry some suitable subgroup of $\mathcal{G}_{F}$. (Alternative formulations of the MLFV hypothesis have also been proposed in [7,-9].)

\subsection{1. $S U(3)_{N} \rightarrow O(3)_{N} \times C P$.}

Assuming that the flavour group acting on the $\mathrm{RH}$ neutrinos is $O(3)_{N}$ rather than $S U(3)_{N}$, implies that $Y_{M}$ must be proportional to $I_{3 \times 3}$. However, this condition alone is not enough to deduce the structure of $Y_{v}$ from the seesaw formula. Full predictivity for this framework is ensured only if we further assume that $Y_{v}$ is real: $Y_{v}^{\dagger}=Y_{v}^{T}$, which follows from imposing CP invariance [5]. In this case, since the Majorana mass term has a trivial structure, all LFV effects stem from the (real) Yukawa coupling matrices giving:

$$
\Delta_{6}=\Delta_{8}^{(1)}=\Delta_{8}^{(2)}=Y_{v} Y_{v}^{T}=\frac{\mu_{L}}{v^{2}} U \mathbf{m}_{v} U^{T} .
$$

The main implication for LFV in this scenario is that the largest entries in the $\Delta$ 's are determined by the heaviest neutrino mass. We refer to [5] for further details.

\subsection{2. $S U(3)_{\ell} \times S U(3)_{N} \rightarrow S U(3)_{\ell+N}$.}

If we assume that $\ell$ and $N$ belong to the fundamental representation of the same $S U(3)$ group, then in a generic basis $Y_{v}$ must be a unitary matrix (and thus it can be always rotated to the identity matrix by a suitable unitary transformation of the $\mathrm{RH}$ neutrinos). This condition, first proposed in [6], also allows to invert the seesaw formula in eq. (7), giving

$$
\Delta_{6}=\frac{v^{2}}{\mu_{L}} U \frac{1}{\mathbf{m}_{v}} U^{T}, \quad \Delta_{8}^{(2)}=\frac{v^{4}}{\mu_{L}^{2}} U \frac{1}{\mathbf{m}_{v}^{2}} U^{\dagger},
$$

while $\Delta_{8}^{(1)}=I_{3 \times 3}$ gives no LFV effects. The choice of a unitary $Y_{v}$ can be phenomenologically interesting because it has been shown that if the $N$ 's belong to an irreducible representation of a non-Abelian group, then $Y_{v}$ is precisely (proportional to) a unitary matrix [10]. Now, models based on non-Abelian (discrete) groups have proved to be quite successful in reproducing the approximate tri-bimaximal [11] structure of the PMNS matrix, so an approximate unitarity of $Y_{v}$ is what is obtained in several cases. This scenario has also the remarkable implication that the largest LFV effects are controlled by the lightest neutrino mass. Other phenomenologically interesting features are discussed in [6].

Let us note at this point that $\mathbf{m}_{v}^{-1}$ appearing in eq. 12 , does not correspond to any combination of the spurions of the high energy theory. Therefore we learn that, contrary to common belief, a MFV high energy Lagrangian can produce, at low energies, operators which are not MFV. A low energy theory following from a MFV high energy theory is guaranteed to be also MFV only under the additional requirement that, when all the spurions are set to zero, the only massless fields are the SM ones.

\subsection{MLFV Operators}

Several MLFV operators can be constructed with the spurions combinations given in eq. (10) or the analogous structures involving also $Y_{e}$, like $\Delta_{8} Y_{e}$, and it is useful to provide at least a partial classification of the most important ones:

\section{On-shell photonic operators.}

They control the radiative decays $\ell \rightarrow \ell^{\prime} \gamma$, and also contribute to $\mu-e$ conversion in nuclei, and to four-leptons processes like $\ell \rightarrow 3 \ell^{\prime}$ decays. Their structure is:

$$
O_{R L}^{(F)}=\bar{\ell}_{\alpha}\left(\Delta_{8} Y_{e}\right)^{\alpha \beta}(\sigma \cdot F) e_{\beta} \cdot H
$$

where $F$ denotes generically the field strength of the $S U(2)_{L} \times U(1)_{Y}$ gauge fields. When these operators are 

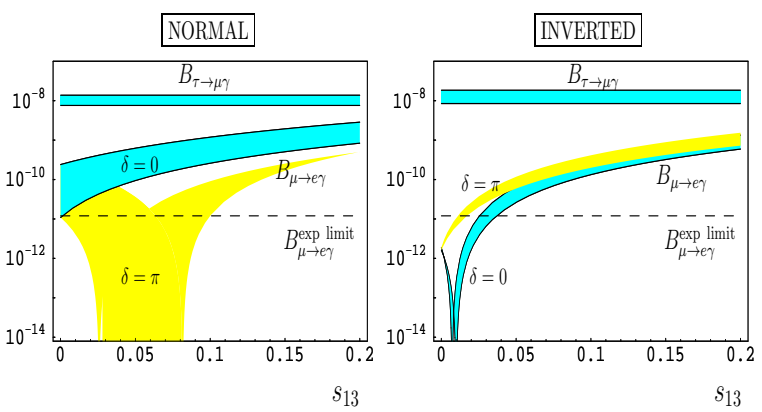

Figure 1: $B_{\tau \rightarrow \mu \gamma}$ and $B_{\mu \rightarrow e \gamma}$ as a function of $\sin \theta_{13}$ for the CP conserving cases $\delta=0, \pi$ and $\Lambda_{N P} \sim 10^{-4}\left(2 v \mu_{L}\right)^{1 / 2}$. The shading corresponds to a lightest $v$ mass in the range $0-0.02 \mathrm{eV}$. (From ref. [5].)

the dominant ones, one can predict quantitative relations between $\mu \rightarrow e \gamma$ and other processes, as for example:

$$
\begin{aligned}
B_{\mu \rightarrow e e e} & \simeq \frac{1}{160} B_{\mu \rightarrow e \gamma} \\
\frac{\Gamma_{\mu T i \rightarrow e T i}}{\Gamma_{\mu T i \rightarrow \text { capt }}} & \simeq \frac{1}{240} B_{\mu \rightarrow e \gamma} .
\end{aligned}
$$

Clearly, in this case the decay $\mu \rightarrow$ er would play an utmost important role in searching for LFV.

\section{Off-shell photonic and contact operators with quarks.}

They can give important contributions in particular to $\mu-e$ conversion in atoms, and have the form:

$$
\begin{aligned}
O_{L L}^{(H)} & =\bar{\ell}_{\alpha} \gamma^{\mu} \tau^{a} \Delta_{8}^{\alpha \beta} \ell_{\beta} \cdot\left(H^{\dagger} \tau^{a} i D_{\mu} H\right), \\
O_{L L}^{(Q)} & =\bar{\ell}_{\alpha} \gamma^{\mu} \tau^{a} \Delta_{8}^{\alpha \beta} \ell_{\beta} \cdot\left(\bar{Q}_{L} \tau^{a} \gamma_{\mu} Q_{L}\right), \\
O_{L L}^{(q)} & =\bar{\ell}_{\alpha} \gamma^{\mu} \Delta_{8}^{\alpha \beta} \ell_{\beta} \cdot\left(\bar{q}_{R} \gamma_{\mu} q_{R}\right),
\end{aligned}
$$

where $\tau^{a}=(1, \vec{\tau})$ with $\vec{\tau}$ the $S U(2)$ matrices and $q_{R}=u_{R}, d_{R}$ denotes the RH quarks.

\section{Four leptons contact operators.}

They can be particularly relevant for $\ell \rightarrow 3 \ell^{\prime}$ decays. The leading operators have the form:

$$
\begin{aligned}
O_{L L}^{(4 \ell)} & =\bar{\ell}_{\alpha} \gamma^{\mu} \tau^{a} \Delta_{8}^{\alpha \beta} \ell_{\beta} \cdot\left(\bar{\ell}_{L} \tau^{a} \gamma_{\mu} \ell_{L}\right) \\
O_{L L}^{(2 \ell 2 e)} & =\bar{\ell}_{\alpha} \gamma^{\mu} \Delta_{8}^{\alpha \beta} \ell_{\beta} \cdot\left(\bar{e}_{R} \gamma_{\mu}, e_{R}\right) .
\end{aligned}
$$

Clearly, in the general case when operators of type 2 . and 3. are not particularly suppressed with respect to the operators in 1 ., searches for $\mu-e$ conversion in nuclei and for LFV decays like $\mu \rightarrow 3 e, \tau \rightarrow 3 \mu$ etc. become equally important than $\mu \rightarrow e \gamma$ to search for LFV. Here we only consider the radiative decays $\ell \rightarrow \ell^{\prime} \gamma$, but a detailed analysis of many others LFV processes within the first MLFV scenario can be found in [12].
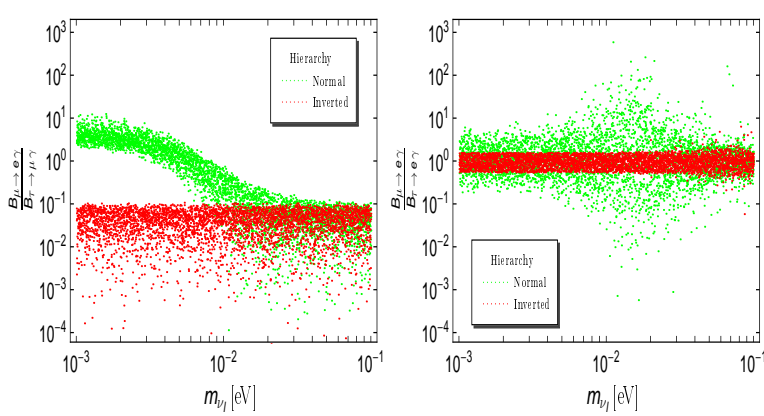

Figure 2: The ratios $\frac{B_{\mu \rightarrow e \gamma}}{B_{\tau \rightarrow \mu \gamma}}$ (left) and $\frac{B_{\mu \rightarrow e \gamma}}{B_{\tau \rightarrow e \gamma}}$ (right) as a function of the lightest neutrino mass. Green-lighter points correspond to normal hierarchy, red-darker points to inverted hierarchy. (From ref. [6].)

\subsection{Phenomenology}

Let us now discuss for the two cases at hand, the dependence of LFV processes on low energy parameters. We concentrate on the radiative decay $\ell_{i} \rightarrow \ell_{j} \gamma$ and on the effects of on-shell photonic operators $O_{R L}^{(F)}$. The relevant LFV structure is $\Delta_{8}$ defined respectively in eqs. (11) and (12), we also assume that all $c_{i}$ in eq. (5) are of $O(1)$. We compare the relevance of different decay channels by means of the normalized branching fractions:

$$
B_{\ell_{i} \rightarrow \ell_{j} \gamma} \equiv \frac{\Gamma_{\ell_{i} \rightarrow \ell_{j} \gamma}}{\Gamma_{\ell_{i} \rightarrow \ell_{j} v_{i} \bar{v}_{j}}} .
$$

When the flavour symmetry $O(3)_{N} \times C P$ is assumed, one observes the pattern $B_{\tau \rightarrow \mu \gamma} \gg B_{\mu \rightarrow e \gamma}\left(\sim B_{\tau \rightarrow e \gamma}\right)$, which is a consequence of the suppression of LFV effects when the lightest neutrinos mass eigenvalues are involved. This is illustrated in Fig. 1] taken from ref. [5] that depicts the normalized branching fractions $B_{\tau \rightarrow \mu \gamma}$ and $B_{\mu \rightarrow e \gamma}$ assuming a NP scale $\Lambda_{N P} \sim 10^{-4} \sqrt{2 v \mu_{L}}$. For a given choice of $\delta=0$ or $\pi$ (corresponding to CP conservation), the strength of the $\mu \rightarrow e$ suppression is very sensitive to whether the hierarchy is normal (NH) or inverted (IH). For $\delta=0$ the present experimental limit on $B_{\mu \rightarrow e \gamma}$ allows large values of $B_{\tau \rightarrow \mu \gamma}$ only for the $\mathrm{IH}$, whereas for $\delta=\pi$, a large region with a sizable $B_{\tau \rightarrow \mu \gamma}$ is allowed only for the $\mathrm{NH}$. Note that the overall vertical scale in this figure depends on both the ratio $\left(v \mu_{L}\right) / \Lambda_{N P}^{2}$ and on the value of the lightest neutrino mass, and that a large hierarchy $\Lambda_{N P} / \mu_{L} \ll 1$ is required to obtain observable effects.

When the assumed flavour symmetry is $S U(3)_{\ell+N}$, the main distinctive feature with respect to the previous case is that, due to the inverse $\mathbf{m}_{v}$ dependence in eq. (12), LFV processes are enhanced when the lighter neutrinos masses are involved. This implies, in particular, a potentially strong enhancement of $\mu \rightarrow e \gamma$ in the $(\mathrm{NH})$ case. This is better highlighted by studying ratios 
of branching ratios for different decay channels, since they simply reduce to ratios of the modulus squared of the corresponding $\Delta_{8}$ entries:

$$
\frac{B_{\ell_{i} \rightarrow \ell_{j} \gamma}}{B_{\ell_{k} \rightarrow \ell_{m} \gamma}}=\frac{\left|\left(\Delta_{8}\right)_{i j}\right|^{2}}{\left|\left(\Delta_{8}\right)_{k m}\right|^{2}} .
$$

Figure 2 (taken from ref.[6]) shows two scatter plots generated with random values for the quantities $\Delta_{8} \sim$ $U \frac{1}{\mathbf{m}_{v}^{2}} U^{\dagger}$, obtained by allowing the neutrino parameters to vary within their (approximate) $2 \sigma$ c.l. experimental intervals [13]. In the left panel we plot, as a function of the lightest mass eigenvalue, the ratio $\frac{B_{\mu \rightarrow e \gamma}}{B_{\tau \rightarrow \mu \gamma}}$, and in the right panel the ratio $\frac{B_{\mu \rightarrow e \gamma}}{B_{\tau \rightarrow e \gamma}}$. Results for the $\mathrm{NH}\left(m_{v_{l}}=m_{v_{1}}\right)$ correspond to the green-lighter points, while the IH $\left(m_{v_{l}}=m_{v_{3}}\right)$ to the red-darker points. From the first panel we see that for $\mathrm{NH}$ and small values of $m_{v_{1}} \lesssim 10^{-2} \mathrm{eV}$ we generically have $B_{\mu \rightarrow e \gamma}>B_{\tau \rightarrow \mu \gamma}$. The enhancement of $B_{\mu \rightarrow e \gamma}$ is obviously due to $\mathbf{m}_{v}^{2}$ appearing in the denominator of $\Delta_{8}$, and can be of a factor of a few. In the limit of $m_{v_{1}} \ll m_{v_{2,3}}$, and using the best fit values of the mixing angles, we have: $\frac{B_{\mu \rightarrow e \gamma}}{B_{\tau \rightarrow \mu \gamma}} \approx$ 7.3 (3.2) for $\delta=0(\delta=\pi)$. When $m_{v_{1}}^{2} \gg \Delta m_{\text {sol }}^{2}$ and $m_{v_{1}} \approx m_{v_{2}}$, the contributions to $\mu \rightarrow e \gamma$ proportional to $\theta_{12}$ suffer a strong GIM suppression, and the decay rate becomes proportional to $\theta_{13}^{2}$. This behavior is seen clearly in Fig. 2 (left) for values of $m_{v_{1}} \approx 10^{-2} \mathrm{eV}$. For $\mathrm{IH}$, in the limit $m_{v_{3}} \ll m_{v_{1,2}}$ and independently of the value of $\delta$ we obtain: $\frac{B_{\mu \rightarrow e \gamma}}{B_{\tau \rightarrow \mu \gamma}} \approx 2 s_{13}^{2}$. Approximately the same result is obtained also in the limit of large masses $m_{v_{i}} \gg \sqrt{\Delta m_{\mathrm{atm}}^{2}}$, which explains why for $m_{v_{1}} \rightarrow 10^{-1} \mathrm{eV}$ the results for $\mathrm{IH}$ and $\mathrm{NH}$ converge.

Results for the ratio of the $\mu$ and $\tau$ radiative decays into electrons are depicted in the right panel in Fig.2 At a glance we see that for both $\mathrm{NH}$ and $\mathrm{IH}$ the $\mu / \tau$ ratios for decays into electrons remain centered around one for all values of $m_{v_{l}}$. Needless to say, since the ratio of normalized branching ratios of other LFV processes like for example $B_{\mu \rightarrow 3 e}, B_{\tau \rightarrow 3 \mu}, B_{\tau \rightarrow 3 e}$ are controlled by the same LFV factors $\Delta_{8}$, they are characterized by a completely similar pattern of enhancements/suppressions.

In view of the ongoing high sensitivity searches for LFV processes [14], besides comparing the rates for different LFV channels, an estimate of the absolute values of the branching fractions is also of primary interest. In the most favorable case, in which $\Delta_{8}$ is a matrix with $O(1)$ entries, a rough estimate gives:

$$
B_{\mu \rightarrow e \gamma} \approx 1536 \pi^{3} \alpha \frac{v^{4}}{\Lambda_{N P}^{4}} .
$$

When compared with the experimental limit $B_{\mu \rightarrow e \gamma}^{\exp }<$ $10^{-11}$ [15] this allows us to conclude that the scale of NP should be rather large: $\Lambda_{N P} \gtrsim 400 \mathrm{TeV}$.

In summary, this second MLFV scenario [6] is characterized by a quite different phenomenology from the first one since it allows the branching fraction $B_{\mu \rightarrow e \gamma}$ to dominate over $B_{\tau \rightarrow \mu \gamma}$ and $B_{\tau \rightarrow e \gamma}$. The enhancement with respect $B_{\tau \rightarrow \mu \gamma}$ that occurs in the NH case does not exceed a factor of a few, but it is parametric in the small values of $m_{v_{1}}$. The strong enhancement with respect to $B_{\tau \rightarrow e \gamma}$ instead is due to accidental cancellations that suppress this process, and that become particularly efficient when $\delta$ is close to zero.

\section{Acknowledgments}

I thank the authors of ref. [5] for permission to include fig. 1 in this review.

\section{References}

[1] R. S. Chivukula, H. Georgi, Phys. Lett. B188, 99 (1987); L. J. Hall, L. Randall, Phys. Rev. Lett. 65, 2939-2942 (1990).

[2] A. J. Buras, P. Gambino, M. Gorbahn, S. Jager and L. Silvestrini, Phys. Lett. B 500 (2001) 161 |arXiv:hep-ph/0007085|.

[3] G. D'Ambrosio, G. F. Giudice, G. Isidori and A. Strumia, Nucl. Phys. B 645, 155 (2002) arXiv:hep-ph/0207036.

[4] S. Weinberg, Phys. Rev. Lett. 43, 1566-1570 (1979).

[5] V. Cirigliano, B. Grinstein, G. Isidori and M. B. Wise, Nucl. Phys. B 728, 121 (2005) [arXiv:hep-ph/0507001].

[6] R. Alonso, G. Isidori, L. Merlo, L. A. Munoz and E. Nardi, JHEP 1106, 037 (2011) [arXiv:1103.5461 [hep-ph]].

[7] S. Davidson and F. Palorini, Phys. Lett. B 642 (2006) 72 |arXiv:hep-ph/0607329].

[8] M. B. Gavela, T. Hambye, D. Hernandez and P. Hernandez, JHEP 0909, 038 (2009) |arXiv:0906.1461 [hep-ph]].

[9] A. S. Joshipura, K. M. Patel and S. K. Vempati, Phys. Lett. B 690, 289 (2010) |arXiv:0911.5618 [hep-ph]].

[10] E. Bertuzzo, P. Di Bari, F. Feruglio and E. Nardi, JHEP 0911, 036 (2009) |arXiv:0908.0161 [hep-ph]].

[11] P. F. Harrison, D. H. Perkins and W. G. Scott, Phys. Lett. B 530 (2002) 167 |arXiv:hep-ph/0202074|; Z. z. Xing, Phys. Lett. B 533 (2002) 85 |arXiv:hep-ph/0204049|.

[12] V. Cirigliano, B. Grinstein, Nucl. Phys. B752, 18-39 (2006). |hep-ph/0601111].

[13] G. L. Fogli, E. Lisi, A. Marrone, A. Palazzo, A. M. Rotunno, Phys. Rev. Lett. 101, 141801 (2008) |arXiv:0806.2649 [hepph]]; |arXiv:0809.2936 [hep-ph]]; T. Schwetz, M. A. Tortola, J. W. F. Valle, New J. Phys. 10, 113011 (2008) arXiv:0808.2016 [hep-ph]]; M. Maltoni, T. Schwetz, PoS IDM2008, 072 (2008) |arXiv:0812.3161 [hep-ph]].

[14] F. Cei [MEG Collaboration], J. Phys. Conf. Ser. 259, 012010 (2010); S. Mihara [MEG Collaboration], Nucl. Phys. A844, 150C-154C (2010); S. Ritt [MEG Collaboration], Nucl. Phys. Proc. Suppl. 162, 279-282 (2006).

[15] M. L. Brooks et al. [MEGA Collaboration], Phys. Rev. Lett. 83, 1521-1524 (1999) |hep-ex/9905013]; M. Ahmed et al. [MEGA Collaboration], Phys. Rev. D65, 112002 (2002) |hepex/0111030 